\title{
Qualification et optimisation d'un compteur dynamique à ogive centrale
}

\author{
par T.M. Pham, J.M. Michel \\ Laboratoire des Ecoulements Géophysiques et Industriels \\ Y. Lecoffre \\ Ylec Consultants
}

\section{INTRODUCTION}

La qualité des liquides joue un rôle fondamental sur l'initiation et le développement de la cavitation. D'un point de vue pratique, les essais menés sur modèles réduits en tunnel hydrodynamique ne sont transposables à l'échelle des prototypes qu'à condition d'assurer des conditions de similitude sur les concentrations de germes actifs [9]. La mesure des germes apparaît à ces deux titres un enjeu majeur aussi bien pour la compréhension fondamentale des phénomènes de cavitation que pour la qualification des moyens d'essais.

Nous pouvons distinguer deux principales techniques de mesure de germes de cavitation : les mesures optiques (holographie, diffusion de la lumière, méthode des phases), et les méthodes dynamiques (mesure par système venturi). La mesure dynamique présente des avantages appréciables par rapport aux méthodes optiques : la mesure des seuls germes actifs, la possibilité de mesurer des germes de taille inférieure à $5 \mu \mathrm{m}$, inaccessibles aux méthodes optiques, le caractère représentatif des échantillons analysés, ainsi que le temps limité de la mesure.
Le principe de la mesure dynamique des germes est le suivant : on soumet l'échantillon liquide à analyser à un champ de dépression donné en le faisant passer dans un venturi. Les germes contenus dans l'échantillon sont alors excités par mise en dépression afin de les transformer en bulles de vapeur détectables. Le niveau de pression minimale est créé au col. Tout germe dont la pression critique est supérieure à cette valeur est activé. Il génère une bulle de cavitation qui, après une phase de grossissement, implose dans une zone de remise en pression, le divergent. Le dénombrement des germes se fait par détection optique ou acoustique. On en déduit les concentrations en germes actifs en rapportant le nombre de germes par seconde au débit traversant le venturi. En répétant cette opération pour différentes pressions au col, on détermine la répartition cumulée des germes en concentration-pression critique. Le concept de venturi de mesure de germes est dû à D. Oldenziel [12]. Il s'agissait de tubes de verre étirés. La détection des germes se faisait en utilisant une barrière optique. Vers 1975, le concept d'Oldenziel a trouvé une opportunité de réalisation industrielle auprès des établissements Neyrtec. Les compteurs venturi à

\section{NOMENCLATURE}

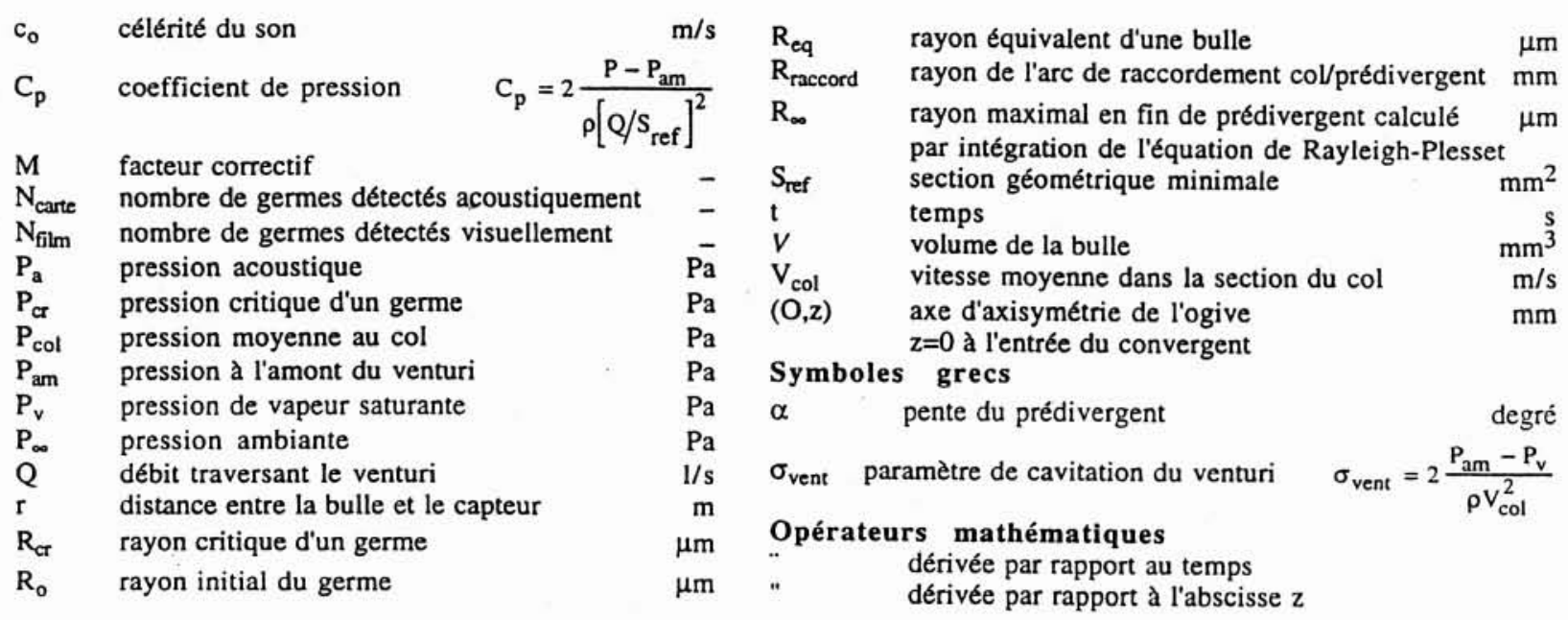


ogive centrale ont été développés par la suite chez Neyrtec (brevet $84 / 180820,1984$ ) pour répondre à un souci de qualité de réalisation et à un désir d'augmenter les débits analysables.

L'objectif de notre étude est de définir une conception de référence du compteur à ogive centrale, la mieux caractérisée possible. L'idée est de s'inspirer de l'appareil préexistant mais de simplifier ses formes ; la modification la plus importante réside dans la forme du conduit extérieur, cylindrique sur toute sa longueur alors que la tuyère du modèle précédent est un cône. Cette géométrie simple, qui est d'autant plus aisée à étudier à la fois sur le plan numérique et expérimental, est ensuite optimisée compte-tenu des contraintes imposées par les interactions entre la cavitation et la couche limite. Une qualification de l'appareil optimisé établit alors ses limites de fonctionnement, et ses performances -précision sur la mesure de la pression critique des germes et de leur nombre. Il est à remarquer que nous avons opté pour une détection acoustique des germes au moyen d'un capteur piézo-électrique placé dans la zone de remise en pression. Aussi, restreindrons-nous notre discussion à la mesure des germes par compteur venturi associé à un système de détection acoustique.

\section{II $\square$ CONCEPTION ET DIMENSIONNEMENT DU VENTURIX}

\subsection{Architecture générale}

L'architecture générale du Venturix est présentée en figure 1. Le capteur hydrodynamique est constitué des éléments suivants :

- Une forme externe cylindrique de diamètre intérieur égal à $20 \mathrm{~mm}$. Cette forme se poursuit jusqu'à la fin du prédivergent. Elle est appelée tuyère.

- Un corps central, appelé ogive, permettant de réaliser un convergent, un col, puis un prédivergent. Les éléments de ce corps central sont :

- un convergent formé par un ellipsoïde de révolution dont le rapport du grand axe au petit axe est de 3/1. Cela permet de réaliser la convergence sans créer de survitesses trop importantes à l'entrée du col ;

- le col formé d'un cylindre d'une longueur de $10 \mathrm{~mm}$ et de diamètre $18 \mathrm{~mm}$;

- le prédivergent formé d'un tronc de cône de demi-angle au sommet égal à $2^{\circ}$. La longueur de ce prédivergent est de $30 \mathrm{~mm}$. La vitesse de l'écoulement à sa sortic est moitié de celle qui existe au col. Le raccordement de la partie cylindrique à ce tronc de cône se fait par un arc de rayon $\mathrm{R}_{\text {raccord }}$ égal à $200 \mathrm{~mm}$.

Le divergent proprement dit se fait sur la tuyère par augmentation du diamètre intérieur. On passe, selon un angle de $2,64^{\circ}$, d'un diamètre de $20 \mathrm{~mm}$ à un diamètre de $26 \mathrm{~mm}$ sur une longueur de $65 \mathrm{~mm}$. La vitesse à la sortie du divergent est environ 5 fois plus faible que la vitesse au col. Pour assurer des conditions d'entrée dans le Venturix aussi homogènes que possibles, et indépendantes des conditions d'écoulement amont, on place à l'amont du capteur :

- un préconvergent d'entrée assurant le passage d'un diamètre de $30 \mathrm{~mm}$ à un diamètre de $20 \mathrm{~mm}$;

- un nid d'abeille de longueur $42 \mathrm{~mm}$, de maille hexagonale $3 \mathrm{~mm}$, situé à $140 \mathrm{~mm}$ en amont du préconvergent.

Ces éléments ne sont pas représentés dans la Figure 1.

Le choix de la longueur du col, ainsi que celui de la section minimale d'écoulement sont en accord avec des études de

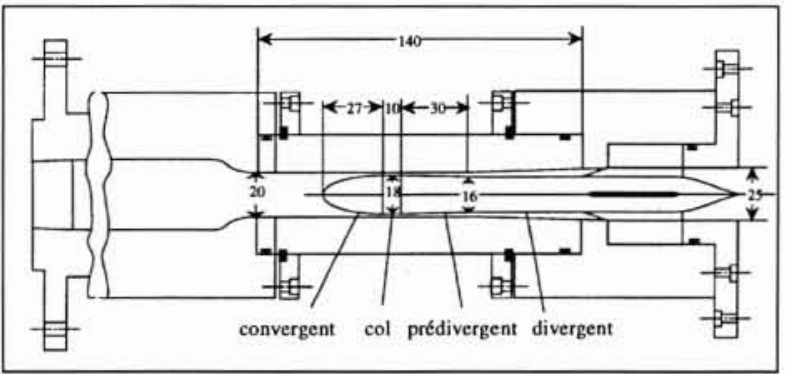

1. Architecture du capteur hydrodynamique.

dimensionnement de tubes venturi antérieurement menées [3][10]. Une étude d'optimisation des formes, portant sur le tracé du col et la conception du prédivergent, est ensuite conduite sur la base de cette architecture générale. Cette étude vise essentiellement à contrôler toutes causes de dysfonctionnement de l'appareil, notamment à prévenir le développement d'une cavitation par poche dans la zone de remise en pression, cause d'un "blocage" de la pression à la pression de vapeur $\mathrm{P}_{\mathrm{v}}(\S 3.1)$. Nous cherchons également à assurer une répartition de pression "optimale" dans la section du col du point de vue développement et détection des germes.

\subsection{Optimisation du tracé du col}

L'objectif de notre étude d'optimisation est de prendre en compte les effets visqueux dans le tracé du col. En effet, le développement des couches limites sur les parois du capteur induit une diminution de la section réelle d'écoulement du fluide par rapport à la section géométrique du venturi, diminution d'autant plus importante que le nombre de Reynolds (i.e. le débit traversant le venturi) est faible. Nous cherchons à créer une distribution de pression constante dans le col pour une vitesse nominale au col de $20 \mathrm{~m} / \mathrm{s}$ (correspondant à un débit de $1,2 \mathrm{l} / \mathrm{s}$ ), compte-tenu du développement des couches limites. La géométrie cylindrique de l'ogive au niveau du col est modifiée en conséquence.

Ce choix de répartition de pression dans le col se justifie ainsi

- On conçoit qu'une répartition de pression optimale du point de vue du développement des germes consiste en une répartition constante.

- Le Venturix doit couvrir une gamme de débits analysables de 0,3 à $3 \mathrm{l} / \mathrm{s}$.

Cela correspond à une plage de vitesses moyennes au col comprises entre 5 et $50 \mathrm{~m} / \mathrm{s}$. Aussi, avons-nous choisi de prendre

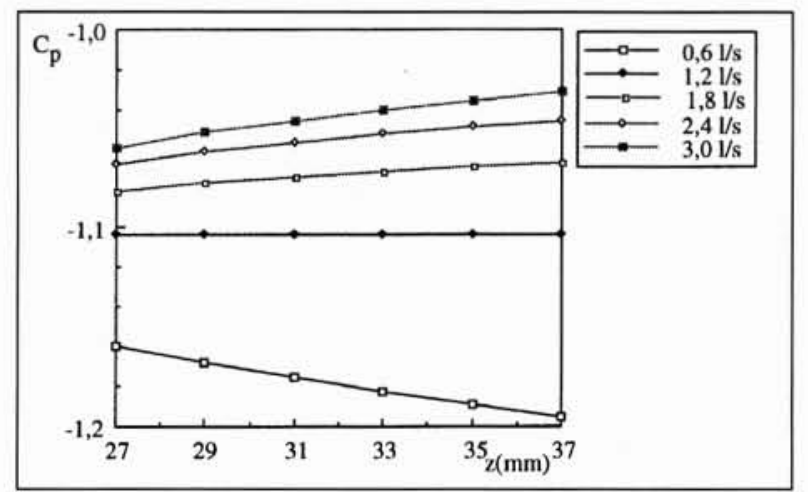

2. Répartition de Cp dans le col - Estimation numérique. 
comme vitesse nominale la valeur intermédiaire de $20 \mathrm{~m} / \mathrm{s}$ afin de minimiser les écarts par rapport à la valeur constante de la pression sur toute la gamme des vitesses.

La procédure suivie est la suivante : le choix de la géométrie générale du Venturix étant arrêté, une modélisation en fluide parfait de l'écoulement dans le capteur par la méthode intégrale aux frontières donne accès au champ de pression interne et en particulier aux répartitions de pression sur les parois du Venturix. Un calcul de couches limites est mené sur la base de ces distributions de pression pour la vitesse de référence de $20 \mathrm{~m} / \mathrm{s}$ au col. Les épaisseurs de déplacement sur les parois de l'ogive et de la tuyère nous donnent la déviation des lignes fluides due aux couches limites. Nous pouvons alors définir la divergence nécessaire pour compenser les effets visqueux et obtenir un plateau de pression au col : une légère divergence du col est ménagée en diminuant le rayon de l'ogive d'une quantité égale à la somme des épaisseurs de déplacement, la géométrie de la tuyère demeurant inchangée. En sortie de col, le rayon de l'ogive est diminué d'environ $0,01 \mathrm{~mm}$. Sur la base de cette nouvelle géométrie, une procédure semblable permet d'estimer les répartitions de Cp le long du col, pour des débits compris entre 0,6 et $3,01 / \mathrm{s}$ (Figure 2). Ainsi, on connait a priori les pressions minimales créées au col sur la gamme de fonctionnement de l'appareil. Des écarts sur les $\mathrm{C}_{\mathrm{pmin}}$ allant jusqu'à $13 \%$ sont observés.

En ce qui concerne la réalisation du Venturix, remarquons que des tolérances de réalisation strictes sont requises afin d'atteindre des précisions dimensionnelles compatibles avec les valeurs des épaisseurs de déplacement qui ont été évaluées. En conséquence, on a décidé de réaliser la tuyère et l'ogive en acier inoxydable.

\subsection{Optimisation de la zone de remise en pression}

\subsubsection{Une conception initiale à réviser...}

Nous avons présenté en § 2.1 la géométrie de la zone de remise en pression qui a été initialement définie. Afin de vérifier que cette géométrie assurait un fonctionnement satisfaisant du dispositif, des essais préliminaires de visualisation de l'écoulement ont été menés au moyen d'une tuyère transparente en plexiglas. Ces essais ont mis en évidence la présence pratiquement systématique d'une poche de cavitation stable se développant dans le prédivergent. La jonction entre la section du col et le prédivergent semble être à l'origine d'une cavitation parasite due au décollement de la couche limite [2], [8]. Cela invalide la conception initiale choisie. Une modification de la conception initiale de l'appareil s'avère indispensable afin de contrôler le risque de décollement de la couche limite dans le prédivergent.

\subsubsection{Contrôle des couches limites par augmentation du taux de turbulence amont}

Deux modifications ont été envisagées : soit définir une géométrie plus progressive du prédivergent en diminuant la pente de la divergence $\alpha$ et/ou en augmentant le rayon $R_{\text {raccord }}$ de l'arc de raccordement à la section du col, soit mettre en place des générateurs de turbulence afin de provoquer la transition à la turbulence et empêcher le décollement laminaire. Une analyse par voie numérique du comportement des couches limites permet d'estimer les répercussions d'une modification de la géométrie du capteur ou d'une augmentation du taux de turbulence Tu : un calcul de couche limite par méthode intégrale (logiciel de calcul AO73 de l'ONERA) est mené dans le convergent et la zone de remise en pression en faisant varier les paramètres $\alpha$, $R_{\text {raccord }}$, Tu, pour des vitesses au col comprises entre 10 et 30 $\mathrm{m} / \mathrm{s}$. La transition à la turbulence est déterminée grâce au critère expérimental d'Habiballah et Arnal [1]. Nous cherchons en particulier à prédire l'existence éventuelle d'un décollement laminaire ainsi que sa position. Notre analyse a conduit à la conclusion qu'une simple modification de la géométrie du prédivergent était inefficace pour contrôler le risque de décollement de la couche limite. En revanche, pour un taux de turbulence de $1 \%$, considérant la géométrie initiale du prédivergent ( $\alpha=1,9^{\circ}$; $\mathrm{R}_{\text {raccord }}=200 \mathrm{~mm}$ ), aucun décollement n'a été prédit sur toute la plage de vitesse considérée. Nous avons décidé de laisser le tracé du prédivergent inchangé et de générer de la turbulence afin de provoquer la transition de la couche limite. Pour définir la géométrie des dispositifs d'excitation de la turbulence, nous avons procédé de manière empirique, en testant diverses formes de générateurs placés à différentes abscisses à l'amont du compteur. La solution choisie consiste à mettre en place deux pièces annulaires usinées sur les parois en contact avec l'écoulement qui agissent localement sur les couches limites : un anneau à profil carré d'épaisseur $2 \mathrm{~mm}$, saillant d'une hauteur de $0,75 \mathrm{~mm}$ par rapport à la paroi est monté en paroi de la conduite d'alimentation du Venturix, à $20 \mathrm{~mm}$ en amont du nez de l'ogive. Un second générateur de turbulence, constitué d'une bague annulaire de $1 \mathrm{~mm}$ d'épaisseur à profil carré, et de $8 \mathrm{~mm}$ de diamètre intérieur, est placé sur le nez de l'ogive. Anticipant quelque peu sur les résultats de notre étude de qualification, nous remarquons que cette solution a permis de faire totalement disparaître le décollement, l'augmentation de la perte de charge du venturi due à la présence des générateurs de turbulence demeurant dans une plage admissible. Ainsi, la mise en œuvre de générateurs de turbulence constitue une alternative très intéressante à l'option généralement adoptée de définir un diffuseur très progressif, solution impliquant des conditions de réalisation mécanique très strictes.

Un prototype du Venturix a été réalisé suivant l'étude de conception présentée ci-dessus. La tuyère du prototype est munie d'un capteur piézo-électrique situé en milieu de prédivergent pour la détection acoustique des germes. Ce capteur est relié au système d'acquisition et de traitement du signal commercialisé par les établissements ACB-Cerg (module électronique du système V.A.G.). Outre ce prototype, ont été fabriquées deux autres tuyères destinées à équiper le Venturix lors d'essais de qualification : une tuyère en acier inoxydable équipée d'une dizaine de prises de pression statique pour la mesure de pression lors d'essais en régime subcavitant, une tuyère en plexiglas permettant la visualisation de l'écoulement et munie également d'un capteur pour la détection acoustique des germes.

Nous présentons dans les paragraphes suivants les résultats des activités expérimentales et numériques qui ont été conduites afin, d'une part, de valider la conception proposée (\$3), d'autre part, de qualifier le système de comptage et de détection acoustique des germes ( $\$ 4$ et 5).

\section{III — VALIDATION DE LA CONCEPTION PROPOSÉE}

\subsection{Contrôle des couche limites et limites de fonc- tionnement}

Le compteur venturi est un appareil qui fonctionne par principe en régime subcavitant. Son régime nominal de fonctionne- 
ment correspond à un écoulement avec bulles voyageuges (ou "séparées") : chaque explosion/implosion de bulles constitue un événement isolé et n'induit pas de perturbation significative du champ hydrodynamique subcavitant. Nous pouvons distinguer deux configurations d'écoulement possibles, causes de dysfonctionnement de l'appareil [3][5] :

- la cavitation par poche attachée due à un décollement laminaire dans le divergent, pour laquelle la cavité qui se développe impose au col une pression égale à la pression de vapeur,

- la cavitation par poches instables (dites "patchs de cavitation").

Lorsque la pression au col est diminuée en deçà d'une valeur limite, un nombre excessif de germes est activé. Leur grossissement de plus en plus important aboutit à la formation de cavités voyageuses (appelées "patchs" de cavitation). L'encombrement de la section d'écoulement par les bulles est tel qu'il y a augmentation globale des pertes de charge et blocage ou saturation ("choking") de l'appareil.

Afin de définir les limites physiques de fonctionnement du prototype construit, une carte des régimes d'écoulement est établie au moyen d'essais de visualisation au travers de la tuyère en plexiglas. Nous présentons en figure 3 les résultats des essais de visualisation effectués sur le venturi équipé du dispositif d'excitation de la turbulence décrit en \$2.3.2. Cette dernière présente la carte des régimes d'écoulement dans le plan $\left(\mathrm{V}_{\mathrm{col}}, \mathrm{P}_{\mathrm{col}}\right) . \mathrm{V}_{\mathrm{col}}$ désigne la vitesse moyenne d'écoulement dans la section du col. Elle est calculée à partir du débit pour une aire d'écoulement correspondant à un entrefer de $1 \mathrm{~mm}$ entre l'ogive et la tuyère. La pression moyenne $\mathrm{P}_{\text {col }}$ régnant dans le col est déterminée à partir de la mesure de la pression à l'amont du venturi $\mathrm{P}_{a m}$ grâce à la relation de Bernoulli.

Ainsi, l'utilisation des générateurs de turbulence pour contrôler les couches limites s'est révélée probante puisque le venturi ne présente aucun décollement, quelle que soit la vitesse d'écoulement. Son fonctionnement est uniquement limité par le risque de saturation au col. La valeur moyenne correspondante du paramètre de cavitation du Venturix limite est égale à $\left(\sigma_{\text {vent }}\right)_{\text {sat }}=0,6$. Des mesures de pression fluctuante à l'aval immédiat de l'anneau monté en paroi de la tuyère en régime subcavitant, pour des débits compris entre 0,3 et $2,1 \mathrm{l} / \mathrm{s}$, ont permis de qualifier les fluctuations de pression induites par les générateurs de turbulence. Elles ont montré que la mise en place de générateurs de turbulence à l'entrée du venturi ne perturbait pas

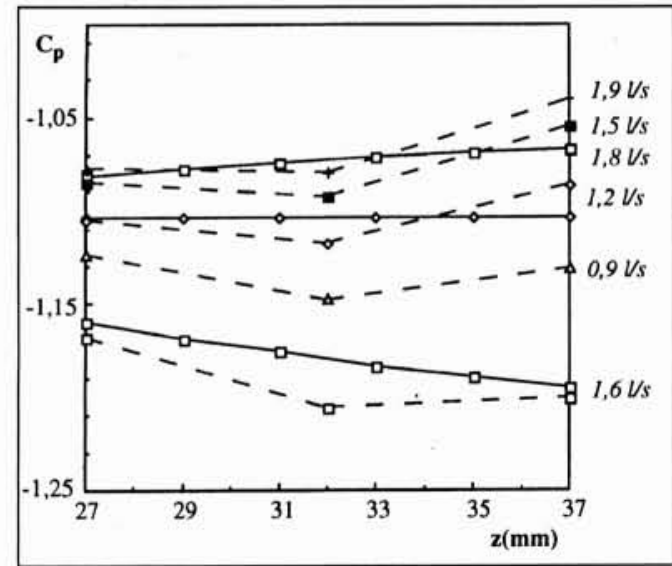

4. Répartition de pression dans le col pour des débits de 0,6 à $1,9 \mathrm{l} / \mathrm{s}$ - Comparaison modélisation (trait plein) / expérimentation (trait pointillé).

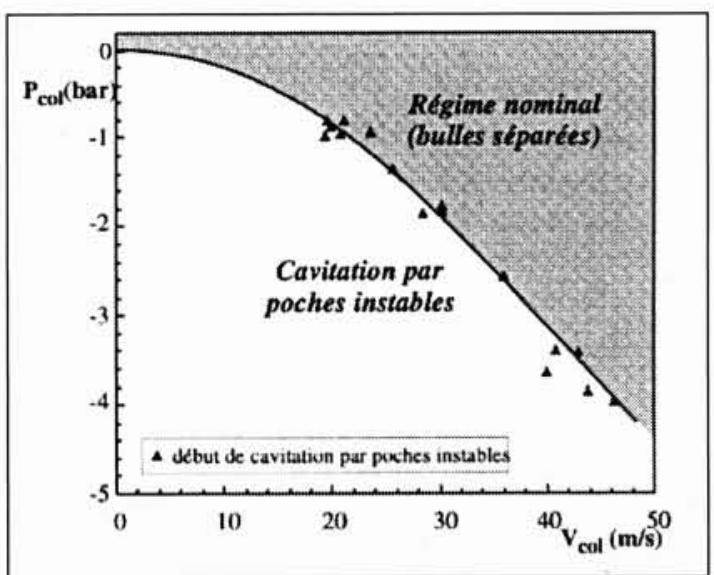

3. Limites de fonctionnement- Carte des régimes d'écoulement dans le Venturix.

la mesure des pressions critiques des germes, ni par une modification de la pression critique des germes par diffusion rectifiée, ni par la mise en résonance ou l'excitation des germes avant la zone de mesure.

\subsection{Distribution de pression dans le venturi - Effet du nombre de Reynolds}

Le présent paragraphe traite de l'étalonnage du Venturix par mesure directe de pression, ainsi que de la comparaison des résultats de mesure avec l'estimation de l'effet du nombre de Reynolds présentée au \$2.2. Cette comparaison vise à valider le tracé de la section du col qui a été proposé.

Les mesures de pression sont réalisées au moyen d'une tuyère en acier inoxydable équipée de 11 prises de pression statique réparties hélicoïdalement le long du cylindre extérieur. Comptetenu du très étroit passage d'écoulement dans la section du col, une attention particulière est apportée à la dimension ainsi qu'à la réalisation des prises. Le diamètre des orifices est de $0,3 \mathrm{~mm}$, sauf pour les prises de pression situées à l'amont et à l'aval du Venturix dont le diamètre est égal à $1 \mathrm{~mm}$. Trois prises de pression sont placées dans la section du col. Des répartitions de pression le long du venturi ont été établies pour des débits allant de $0,6 \mathrm{l} / \mathrm{s}$ à $1,9 \mathrm{l} / \mathrm{s}$.

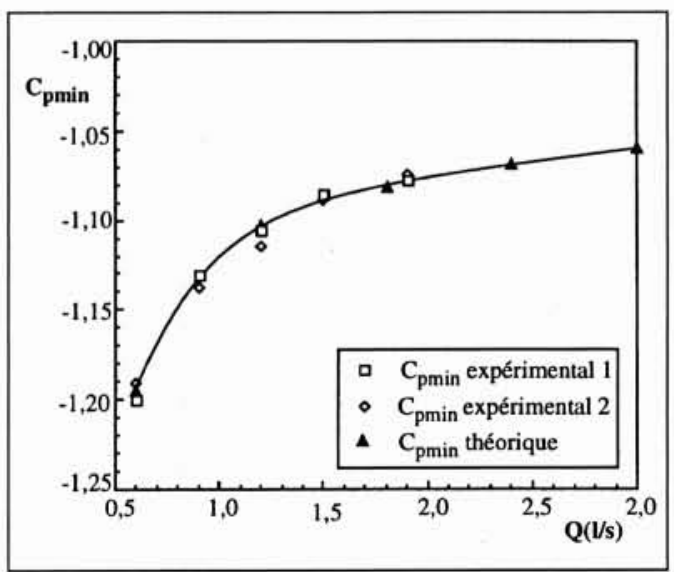

5. Valeur minimale du $C_{p}$ imposé au col en fonction du débit Comparaison modélisation / expérimentation. 
La figure 4 donne les répartitions de pression dans le col correspondantes, comparées aux résultats issus de notre estimation numérique. Les $\mathrm{C}_{\mathrm{pmin}}$ théoriques issus de la modélisation numérique et les $\mathrm{C}_{\mathrm{pmin}}$ déterminés expérimentalement lors de deux campagnes d'essais sont également présentés dans la Figure 5.

D'après les figures 4 et 5 , les résultats de mesure dans le col présentent des ordres de grandeur identiques et des tendances analogues à ceux prévus par la modélisation : pour $\mathrm{Q}=0,9 \mathrm{l} / \mathrm{s}$, on observe que la pente mesurée est faible, avec possibilité de changement de signe : le plateau de pression est obtenu pour $\mathrm{Q} \cong 0,9 \mathrm{l} / \mathrm{s}$ et non pas pour $\mathrm{Q}=1,2 \mathrm{l} / \mathrm{s}$, valeur nominale du débit pour laquelle une pression constante au col était recherchée. Les écarts relatifs observés entre les résultats expérimentaux et les résultats numériques ne sont en aucun cas supérieurs à $2 \%$. Les valeurs minimales de $\mathrm{C}_{\mathrm{p}}$ mesurées dans le col corroborent bien l'analyse théorique et numérique de l'effet des couches limites. Ainsi, le bon accord des résultats expérimentaux avec l'estimation des pressions que nous avions faite valide l'analyse ayant servi au dimensionnement de l'appareil. De plus, la très faible dispersion et la bonne reproductibilité des résultats expérimentaux montrent qu'un étalonnage des venturi par mesure directe des pressions est possible.

\section{IV ■ DÉTECTION ACOUSTIQUE ET COMPTAGE DES GERMES}

\subsection{Dynamique des germes et détection acoustique}

L'objectif est d'estimer les performances théoriques du Venturix quant à la détection acoustique des germes. Il s'agit d'appréhender l'évolution dynamique des germes de cavitation dans le Venturix par une approche numérique, de l'interpréter du point de vue signal acoustique généré. En particulier, nous cherchons à estimer l'influence relative du confinement sur la détection acoustique des nuclei suivant la gamme de dimensions envisagée.

\subsubsection{Modèle numérique}

Considérons une bulle de gaz modélisant un germe de cavitation, initialement sphérique, de rayon $R_{0}$, en équilibre dans les conditions de pression amont et qui pénètre dans le venturi. Nous cherchons à déterminer son évolution ultérieure dans le capteur selon les conditions de pression amont, la pression minimale au col, son rayon initial. Nous faisons l'hypothèse que le liquide est incompressible, qu'il n'existe pas de vitesse de glissement entre la bulle et le liquide, que les transferts de masse à travers l'interface de la bulle sont négligeables, que le gaz contenu dans la bulle a un comportement isotherme et que la pression appliquée à la bulle est égale à la pression dans le liquide en son absence, en un point confondu avec son centre. Jusqu'à l'entrée du col, les bulles sont dans leur phase initiale de grossissement. Elles restent très petites, comparées au passage d'écoulement. Elles peuvent être supposées comme sphériques et en milieu infini : l'équation de Rayleigh-Plesset est intégrée par une procédure de Runge-Kutta d'ordre 4. Dans la section du col, les bulles ont atteint leur taille maximale. Elles sont confinées entre les parois du cylindre et de l'ogive. Afin d'appréhender cet effet de confinement, nous considérons un problème physique plus simple : la bulle est transportée par un écoulement unidimensionnel délimité par deux plaques planes parallèles d'entrefer variable. Le centre de la bulle est assujetti à demeurer dans le plan méridien de l'entrefer. Un suivi de l'interface de la bulle par application de la méthode intégrale aux frontières est effectué. De plus amples informations sur le modèle numérique utilisé sont fournies en [7][13].

\subsubsection{Cas de calculs considérés - Résultats}

Les cas traités sont présentés dans le Tableau 1.

\begin{tabular}{|c|c|c|c|c|c|c|}
\hline & $R_{o}(\mu \mathrm{m})$ & $P_{\infty \infty}(B a r)$ & $P_{\min }(P a)$ & $P_{c}(P a)$ & $R_{c}(\mu \mathrm{m})$ & $R_{\max }(\mu \mathrm{m})$ \\
\hline 1 & 10 & 1,9278 & 0 & 831 & 65 & 620 \\
\hline 2 & 20 & 1,9278 & 0 & 1771 & 181 & 740 \\
\hline 3 & 50 & 1,9278 & 0 & 2164 & 706 & 955 \\
\hline 4 & 100 & 1,9378 & 1000 & 2252 & 2000 & 1100 \\
\hline
\end{tabular}

Tableau 1. - Cas de calcul traités.

$R_{c}$ désigne le rayon critique du germe, et $P_{c}$ sa pression critique. On fait également figurer dans ce tableau les rayons maximaux des germes, notés $\mathrm{R}_{\max }$, calculés par intégration de l'équation de Rayleigh-Plesset portant sur la totalité du domaine de calcul. Cette approche simple permet une première appréhension du comportement des germes dans le capteur. Ainsi, les cas de calcul choisis illustrent deux situations typiques :

$$
\begin{array}{ll}
\text { - Cas } n^{\circ} 1,2,3: P_{\min }<P_{c} R_{\max }>R_{c} \\
\text { - Cas 4: } & P_{\min }<P_{c} R_{\max }<R_{c}
\end{array}
$$

\subsubsection{Dynamique et acoustique de bulles dans le venturi}

Les Figures 6 et 7 illustrent l'évolution du volume (présenté sous forme adimensionnelle par le rapport $\mathrm{R}_{\mathrm{eq}} / \mathrm{R}_{0}$ et de l'interface des bulles dans le venturi, pour les cas de calcul 1 et 4 . $R_{e q}$ désigne le rayon équivalent de la bulle, c'est-à-dire le rayon qu'aurait une bulle sphérique d'égal volume. Pour une description détaillée de la dynamique des bulles dans le Venturix nous référons le lecteur à [13][14]. Nous nous intéressons plus particulièrement à la phase d'implosion des germes (nous nous limitons à $\mathrm{z} \in[38,43 \mathrm{~mm}])$ et voulons estimer la pression acoustique développée lors du collapse de bulles de différentes tailles au niveau du capteur piézo-électrique. La pression acoustique s'exprime par: $P_{a}(r, t)-P_{-}(t)=(\rho / 4 \pi \cdot r) \cdot \ddot{V}\left(t-r / c_{o}\right)$

Elle est proportionnelle à la dérivée seconde temporelle du volume $\ddot{V}(\mathrm{t})$.Compte tenu du fait que la vitesse d'écoulement est pratiquement constante sur la courte portion du prédivergent considérée, $\ddot{V}(\mathrm{t})$ est proportionnelle à la dérivée spatiale $\mathrm{V} "(\mathrm{z})$.

Il se trouve que dans la limite du domaine de calcul effectué les courbes représentatives de V(z) peuvent être approchées par des arcs de parabole. Les valeurs moyennes des dérivées secondes spatiales V"(z) sont déduites des équations des paraboles approchant les courbes $\mathrm{V}(\mathrm{z})$. Leurs valeurs comparées sont récapitulées dans le Tableau 2.

\begin{tabular}{|l|c|c|c|}
\hline & R-P & méthode intégrale & écart R-P/m.intégrale \\
\hline 1 & 0,480 & 0,153 & 0,327 \\
\hline 2 & 0,604 & 0,221 & 0,383 \\
\hline 3 & 0,970 & 0,309 & 0,661 \\
\hline 4 & 1,192 & 0,393 & 0,799 \\
\hline
\end{tabular}

Tableau 2. - Valeurs moyennes des valeurs absolues des dérivées secondes du volume par rapport à z (en $\mathrm{mm}$ ). 


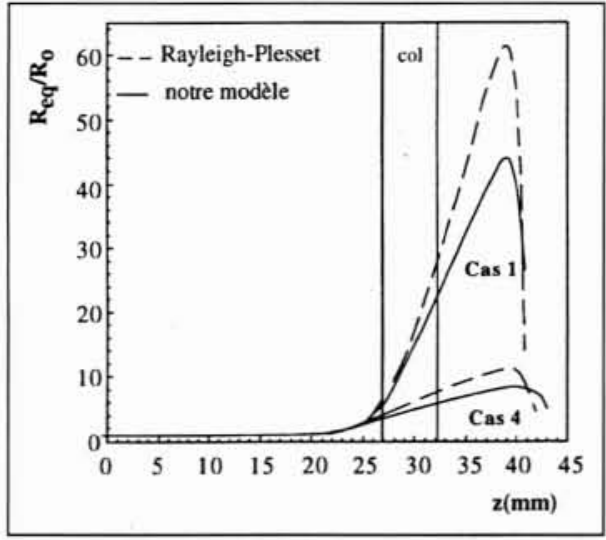

6. Rapport $R_{e q} / R_{o}$ en fonction de l'abscisse $z$ pour cas $n^{\circ} 1$ et $n^{\circ} 4$.

Deux mécanismes antagonistes se dégagent :

1 - Le niveau de bruit généré est croissant avec le volume du germe.

2 - Plus une bulle est grande, plus son collapse est amorti par la présence des parois.

Cependant, cet "amortissement" est insuffisant pour inverser la tendance induite par le mécanisme 1.

Il apparaît que l'acoustique de bulles dans le Venturix présente les mêmes tendances qu'en milieu infini : le bruit émis lors du collapse est d'amplitude d'autant plus importante que le germe dont est issue la bulle est de grande dimension. Cette conclusion est contraire à celles proposées par Ligneul et Bovis [11] d'après lesquelles les germes de rayons importants ne pouvaient pas produire un bruit détectable lors de leur implosion. Ainsi, d'après l'analyse théorique ci-avant, les germes de grande dimension $\left(\mathrm{R}_{\mathrm{o}} \geq 50 \mu \mathrm{m}\right)$ émettent lors de leur implosion un signal acoustique suffisant pour être détectés, sinon supérieur à celui induit par les plus petits nuclei. Ce fait est mis en évidence pour un cas particulièrement "litigieux" quant à la possibilité de détection des germes (cas $\left.n^{\circ} 4\right)$. La détection acoustique des germes dans un compteur venturi n'apparaît pas poser de problème de principe.

\subsection{Etude du fonctionnement du compteur par ciné- matographie rapide}

Une analyse détaillée des phénomènes hydrodynamiques dans le venturi par cinématographie rapide est menée. Nous cherchons plus particulièrement à corréler le nombre d'événements cavitants ayant effectivement lieu dans le venturi au nombre de germes comptés. A cette fin, nous avons réalisé des films rapides au moyen d'une caméra Photec de cadence maximale égale à 10000 images/s en pleine image. Sept films rapides ont été pris pour différents points de fonctionnement du Venturix et dépouillés image par image, pour des nombres de cavitation compris entre 0,82 et 1 .

La caméra Photec est dotée d'un système de double "topage" (marquage de la pellicule au moyen de deux diodes) :

- un "topage" piloté par une source interne fournissant une base de temps : la diode marque le film à une fréquence de 100 ou de $1000 \mathrm{~Hz}$;

- un "topage" pouvant être piloté par une source externe. La source externe choisie dans le cas présent est la sortie analo-

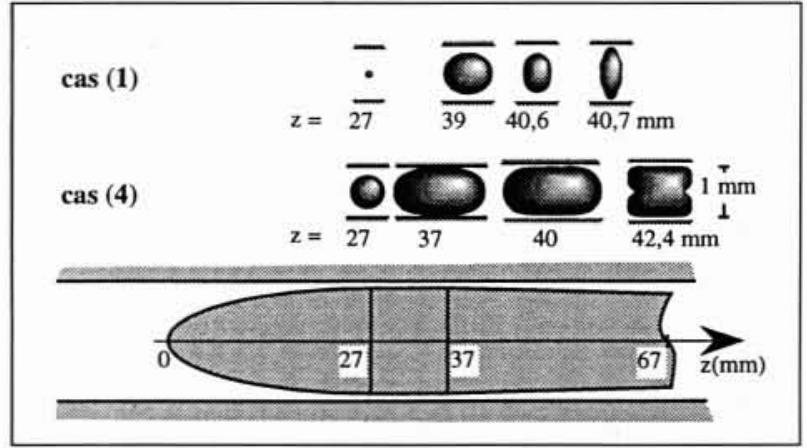

7. Evolution le long du venturi de l'interface des germes pour les cas $n^{\circ} 1$ et $n^{\circ} 4$.

gique de la carte électronique du système de comptage. Le système électronique d'acquisition et de traitement du signal qui équipe le Venturix transforme les signaux oscillants amortis issus du capteur piézo-électrique en une série de créneaux normalisés distincts comptabilisables. La diode impressionne la pellicule durant la durée de chaque créneau. Ceci permet de visualiser "simultanément" (modulo la distance entre les diodes et l'objectif de la caméra) les phénomènes hydrodynamiques dans le venturi et le signal créneau issu de la carte de comptage.

\subsubsection{Corrélation entre le nombre d'événements cavi- tants et le nombre de germes comptés}

Pour chaque film et nombre de cavitation correspondant, on définit un facteur de corrélation moyen qui relie le nombre total de germes dénombrés par la carte au nombre total de germes estimé visuellement :

$$
\left[\frac{\mathrm{N}_{\text {carte }}}{\mathrm{N}_{\text {film }}}\right]_{\text {moyen }}=\frac{1}{\mathrm{M}} \cdot\left(\sum_{\text {séquences }}^{\sum \mathrm{N}_{\text {carte }}} / \sum_{\text {séquences }} \mathrm{N}_{\text {film }}\right)
$$

$M$ est un coefficient multiplicateur qui tient compte du fait que le champ de vision offert par la caméra ne permet pas de voir la totalité de l'écoulement.

Il est déterminé empiriquement et est compris entre 1,1 et 1,85 selon le film considéré. L'évolution du facteur de corrélation en fonction du paramètre de cavitation $\sigma_{\text {vent }}$ est présentée dans la figure 8.

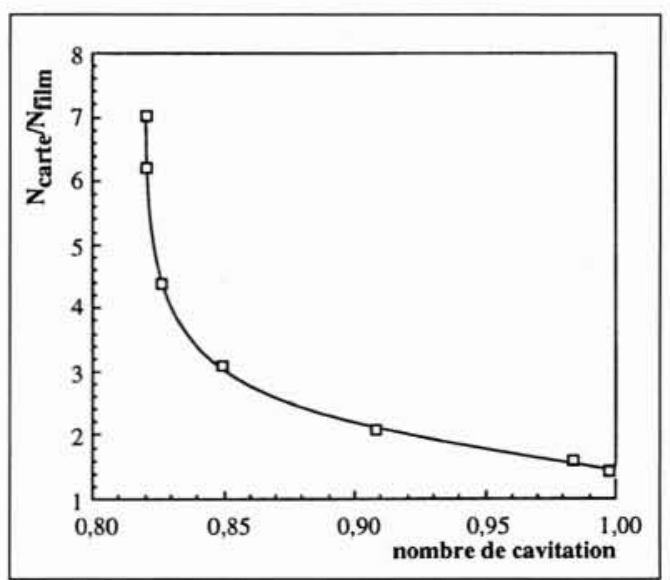

8. Facteur de corrélation en fonction de $\sigma_{\text {vent }}$. 


\subsubsection{Interprétations des résultats}

Au vu des résultats présentés dans la figure 8 , il apparait que le compteur venturi surcompte le nombre de germes de cavitation jusqu'à un facteur 7 .

Le paramètre de cavitation du venturi $\sigma_{\text {vent }}$ doit être supérieur à environ 0,9 pour que le facteur de corrélation demeure dans une marge acceptable de $[1,0 ; 2,0]$, ce qui constitue une limitation assez stricte comparée à $\left(\sigma_{\mathrm{vent}}\right)$ sat $=0,6$. Ce phénomène de surcomptage des germes est à rapprocher de l'analyse des phénomènes dans le venturi. L'examen des films rapides a en effet établi l'existence de deux dynamiques de bulles distinctes suivant la position des points de fonctionnement considérés par rapport à la limite de fonctionnement du Venturix :

- Pour des points de fonctionnement relativement éloignés de la limite, les bulles grossissent jusqu'à une taille millimétrique et implosent de manière assez individuelle.

- Pour des points de fonctionnement proches de la limite, la taille maximale des bulles peut atteindre le centimètre et des déviations très importantes par rapport au comportement précédent ont lieu. Les deux principaux cas de figure observés sont les suivants :

- formation de bulles toriques : la bulle acquiert au cours de son explosion la forme d'un tore d'axe de symétrie normal aux parois solides. L'implosion de la bulle torique s'accompagne de la formation d'un "nuage blanchâtre" visible sur les images qui semble témoigner de la désagrégation du tore en de nombreux fragments. La formation de bulles toriques près d'une paroi solide est rapportée par de nombreux auteurs [4][15]. Leurs travaux ont établi le caractère fortement instable de telles structures.

- scissions de bulles : la bulle lors de son implosion se scinde en plusieurs plus petites bulles qui implosent par la suite de façon non simultanée. Cette observation est en accord avec notre modélisation du comportement des bulles dans le Venturix, ainsi qu'avec des études numériques et expérimentales antérieurement menées concernant l'implosion d'une bulle centrée entre deux parois solides [13],[14],[7].

Des phénomènes de rebonds de bulle ont également été observés. Ainsi, le surcomptage constaté s'expliquerait essentiellement par le fractionnement des bulles qui se produit lorsque $\sigma_{\mathrm{vent}}$ est diminué en deçà d'une valeur limite. Ce phénomène est étroitement lié au confinement des bulles entre deux parois solides.

\section{V $\square$ CONCLUSIONS ET PERSPECTIVES}

Les principales conclusions de notre étude sont les suivantes : - Le Venturix a été conçu et réalisé selon une étude de dimensionnement et d'optimisation des formes visant à prévenir tout décollement laminaire dans la zone de remise de pression et à assurer une répartition de pression constante pour une vitesse nominale au col égale à $20 \mathrm{~m} / \mathrm{s}$. Les innovations essentiellement apportées concernent la compensation des effets visqueux dans la section du col, ainsi que l'utilisation de dispositifs d'excitation de la turbulence pour éviter le décollement laminaire dans le divergent. Suite à cette étude de dimensionnement, un prototype a été fabriqué suivant un cahier des charges strict comptetenu de la conception "très fine" du capteur hydrodynamique.

- La conception retenue a alors été validée par des essais de qualification.
Des essais de visualisation par cavitation de l'écoulement sous éclairage stroboscopique ont montré l'efficacité des générateurs de turbulence quant au contrôle des couches limites. Elles ont permis d'établir les limites de fonctionnement physique de l'appareil. Nous avons de surcroît soigneusement examiné l'éventualité d'une modification de la pression critique des germes par diffusion rectifiée, de leur mise en résonance ou de leur excitation avant la zone de mesure. Des mesures de pression statique le long du venturi se sont révélées être en accord avec l'estimation qui a été faite des répartitions de pression. Cet accord valide le tracé du col qui a été retenu.

Elles ont en outre mis en évidence la possibilité d'étalonner les venturi compteurs de germes par mesure directe des répartitions de pression interne.

- La possibilité théorique de détecter acoustiquement les germes a été discutée et aucune limitation en taille de germes n'a été trouvée. En revanche, l'analyse par cinématographie rapide du fonctionnement du venturi a mis en évidence un surcomptage des germes en deçà d'une valeur limite du paramètre du venturi que nous avons estimée à environ 0,9 . Cette limitation est principalement liée à un phénomène de fractionnement des bulles dans le venturi.

Cette étude a été financée par la Direction des Recherches, Etudes et Techniques dans le cadre du contrat DRET 93-145.

\section{RÉFÉRENCES}

[1]. D. Arnal, M. Habtball.ah, E. Coustols : 1984, "Théorie de l'instabilité laminaire - critères de transition en écoulements bidimensionnel et tridimensionnel", La Recherche Aérospatiale 2.

[2], V.H. ARAKERI, A.J. Acosta : 1973, "Viscous effects on the inception of cavitation on axisymmetric bodies", Part one and Part two, Journal of Fluids Engineering, Vol. no. 95, pp.519-527.

[3]. L. D'Agostino, A.J. AcostA : 1991, "A cavitation susceptibility meter with optical cavitation monitoring", Part one and Part two, Journal of Fluids Engineering, Vol. no. 113, pp.261-277.

[4]. J.R. BLAKE, D.C. GiBson : 1987, "Cavitation bubbles near boundaries", Ann. Rev. Fluid Mech., Vol. no. 19, pp.99-123

[5]. L. BRiAnçon-MArjollet : 1987, "Couche limites, cavités en interaction", Thèse, Grenoble.

[6]. E. CANOT, J.L. ACHARD : 1991, "An overview of boundary integral formulations for potential flows for fluid-fluid systems", Arc. Mec., Warzawa, Vol. no. 43 , pp. $453-498$

[7]. G.L. CHAнINE : 1982, "Experimental and asymptotic study of nonspherical bubble collapse", Appl. Scient. Res., Vol. no. 45, pp.187-197.

[8]. J.P. FRANC, J.M. MICHEL : 1985, "Attached cavity and the boundary layer : experimental investigation and numerical treatment", Journal of Fluid Mechanics, Vol. no. 154, pp.63-90

[9]. J.W. HoLL, G.F. WisLicenus : 1961, "Scale effects of cavitation phenomena", Trans ASME Journal of Basic Engineering, pp.385-395

[10]. Y. LeCOFrre, J. MARCOZ : 1978, "Separate bubble cavitation tests, control-method, nuclei measurement by venturi tube", La Houille Blanche, no. 5 . [11] P. Ligneul, A. Bovis : 1986, "The use and limitations of the venturi tube as a cavitation susceptibility meter", International Symposium on cavitation in Sendai, Japan.

[12].D.M. OLDENZIEL : 1982, "A new instrument in cavitation research : the cavitation susceptibility meter", Journal of Fluids Engineering, Vol. no. 104, pp.136-142.

[13].T.M. Pнам : 1995 , "La mesure des populations de germes de cavitation : qualification et optimisation d'un compteur dynamique à ogive centrale", Thèse, Grenoble.

[14].T.M. Pham, J.M. Michel, Y. LeCofFre : 1995, "Development of a new type of CSM : Design concepts and investigation of performance characteristics", International Symposium on cavitation, CAV'95, Deauville, France.

[15]. A. Vogel, W. Lauterborn, R. Timm : 1989, "Optical and acoustic investigations of the dynamics of laser-produced cavitation bubbles near a solid boundary", Journal of Fluid Mechanics, Vol. no. 206, pp.299-338. 